\title{
Supplement to "A Variational EM Method for Mixed Membership Models with Multivariate Rank Data: an Analysis of Public Policy Preferences"
}

\author{
Y. Samuel Wang and Elena A. Erosheva
}

March 28, 2017

\section{Overview}

This supplement presents a direct comparison of the variational procedure proposed in ? to the existing MCMC procedure of Gormley and Murphy [2009]. To compare estimates and computational time, we consider the 1997 Irish Presidential Election data, the same data that were analyzed with MCMC procedures by Gormley and Murphy [2009] $]^{1}$. For a complete description of the assumed model and notation, we refer readers to Section 5 of the main article.

\section{Data Considered}

Irish Elections utilize a Single Transferable Vote system in which voters cast a rank ordering of their preferred candidates; the candidate with the least first-place votes is eliminated and their votes are redistributed to the voters' next preferred candidate until a majority is reached. We consider exit poll data from 1997 Irish Presidential Election ${ }^{2}$. In the exit poll data, 2,498 voters ranked their preferred candidates among the five individuals running for president. Mary Banotti and Mary McAleese were supported by the two largest parties in Ireland, while Derek Nally, Adi Roche and Rosemary Scallon ran as independents. For a full description of the data we refer readers to Marsh and Mitchell [1999]. In the full election, McAleese received $45.2 \%$ of the first place votes, Banotti received $29.3 \%$, Scallon received $13.8 \%$, Roche received $7.0 \%$ and Nally received $4.7 \%$. The top 10 most popular responses recorded in the exit poll are listed in Table 1. Using a mixed membership model, we seek to identify and characterize latent voting blocs within the Irish electorate.

Table 1: Ten most popular exit poll responses for the 1997 Irish Presidential Election.

\begin{tabular}{lc}
\hline Ballot & Frequency \\
\hline McAleese & 277 \\
McAleese, Scallon & 134 \\
Banotti & 122 \\
McAleese, Banotti & 61 \\
Scallon & 51 \\
Scallon, McAleese & 47 \\
McAleese, Banotti, Roche, Scallon, Nally & 41 \\
McAleese, Banotti, Roche, Nally, Scallon & 39 \\
Banotti, Nally & 38 \\
McAleese, Roche & 38 \\
\hline
\end{tabular}

\section{Computational Effort Comparison}

The original MCMC analysis does not explicitly fit the Dirichlet membership parameter $\alpha$, but instead holds $\alpha$ fixed while sampling the other parameters. Gormley and Murphy [2009] do, however, fit models for several

\footnotetext{
${ }^{1}$ This comparison was originally presented at the Joint Statistical Meetings 2015.

${ }^{2}$ The data was collected by Landsdowne Market Research and is maintained in an archive by Prof. Michael Marsh at Trinity College Dublin.
} 
values of $\alpha$ and show that changing the choice of $\alpha$ (but keeping all elements equal) does not significantly affect the estimates $\theta$. For the remainder of this document, we will use "GM model" to refer to the model from Gormley and Murphy [2009] fit with $\alpha=\{.1, .1, .1, .1\}$.

We first compare computational effort by running a variant of the variational EM method ${ }^{3}$. To make a direct comparison, we also fix the parameter $\alpha$ to $\{.1, .1, .1, .1\}$, and only estimate $\phi, \delta$ and $\theta$. We also set the number of sub-populations $K=4$ as in the GM model. Because McAleese and Banotti were the clear front runners, for each random restart, we initialize two blocs with large support parameters for each of the candidates and use random draws from a Dirichlet distribution to set the support parameters for the remaining two subgroups. We use the two step initialization procedure described in ?, Section 6.4.

Table 2 reports the results from the 20 variational EM method fits and the MCMC procedure for 50,000 iterations (10,000 burn-in iterations and 40,000 samples thinned every 100th iteration $)^{4}$. We can see that a single variational EM run is roughly 140 times faster than a single MCMC run when estimating the same set of parameters. This, however, does not account for random restarts and the multi-modality of the ELBO.

We use the following $\chi^{2}$ statistic to measure goodness-of-fit [Cohen and Mallows, 1983, Erosheva et al., 2007]:

$$
\chi^{2}=\sum_{\sigma: o_{\sigma} \geq 5} \frac{\left(o_{\sigma}-e_{\sigma}\right)^{2}}{e_{\sigma}}
$$

Here, $\mathrm{o}_{\sigma}$ and $\mathrm{e}_{\sigma}$ are the observed and expected counts for a specific rank ordering of the candidates $\sigma$, where $\sigma$ may be an incomplete ranking with less than 5 candidates. Since there are 150 possible responses (including incomplete rankings) and some of these observed rankings appear very infrequently, we truncate the $\chi^{2}$ statistic to only measure the fit of rankings which are observed 5 or more times, although all observed rankings are used when fitting the model. This cutoff includes 113 of the 150 possible responses patterns and 2214 of the 2498 observed responses.

Despite the multi-modality, we note that 14 of the 20 runs result in a stationary point with a better goodness-of-fit than the GM model; so even a single run is generally sufficient for finding a reasonable model (although multiple initializations are still recommended). In addition, each run could be easily computed in parallel.

\begin{tabular}{|c|c|c|}
\hline & Single MCMC Run & Variational EM (mean of 20 batches) \\
\hline Run-time $(\mathrm{sec})$ & 647.3 & 4.5 \\
\hline Truncated $\chi^{2}$ & 1594 & 1469 \\
\hline
\end{tabular}

Table 2: Comparison of run-time and goodness of fit between existing MCMC method and variational EM when $\alpha$ is fixed.

\section{Goodness of Fit Comparison}

We now use the full the variational EM method (including direct estimation of $\alpha$ ). When $\alpha$ varies, we observe an increased number of local modes, so we use 40 random restarts and select the stationary point with the largest lower bound. On average, each of the runs required 15.6 seconds and could have been computed completely in parallel. The model with the highest ELBO results in a $\chi^{2}=842$ compared to the GM model $\chi^{2}=1594$. The estimated parameters are shown in Figure 1 and Table 3.

Aside from the goodness of fit improvement, we see that explicitly estimating $\alpha$ also results in substantiative interpretation changes. In the GM model, we see two subgroups with large support for the eventual winner Mary McAleese. Indeed McAleese received roughly half of the first place votes, so 2 of the 4 (equally sized) subgroups are needed to appropriately capture her full popularity in the GM model. From the estimated support parameters, we might conclude that there was one block of voters who supported Mary McAleese solely and another block of voters who primarily supported Mary McAleese but also had strong support for Scallon as a second choice. However, in the variational EM method, allowing for a larger subgroup allows the remaining subgroups to capture other less salient "voting blocs" which are not captured

\footnotetext{
${ }^{3}$ Each of the experiments presented were run on a quad-core $2.4 \mathrm{Ghz}$ laptop.

${ }^{4}$ The MCMC code was graciously provided by Claire Gormley
} 
when $\alpha$ is fixed. In the variational EM estimates there is only 1 block with a strong preference for McAleese; however, this block has an estimated relative frequency of $45 \%$. This large relative frequency of this subgroup allows the model to fully capture McAleese's popularity while also capturing other Nally/Scallon and Scallon/Roche voting blocks.
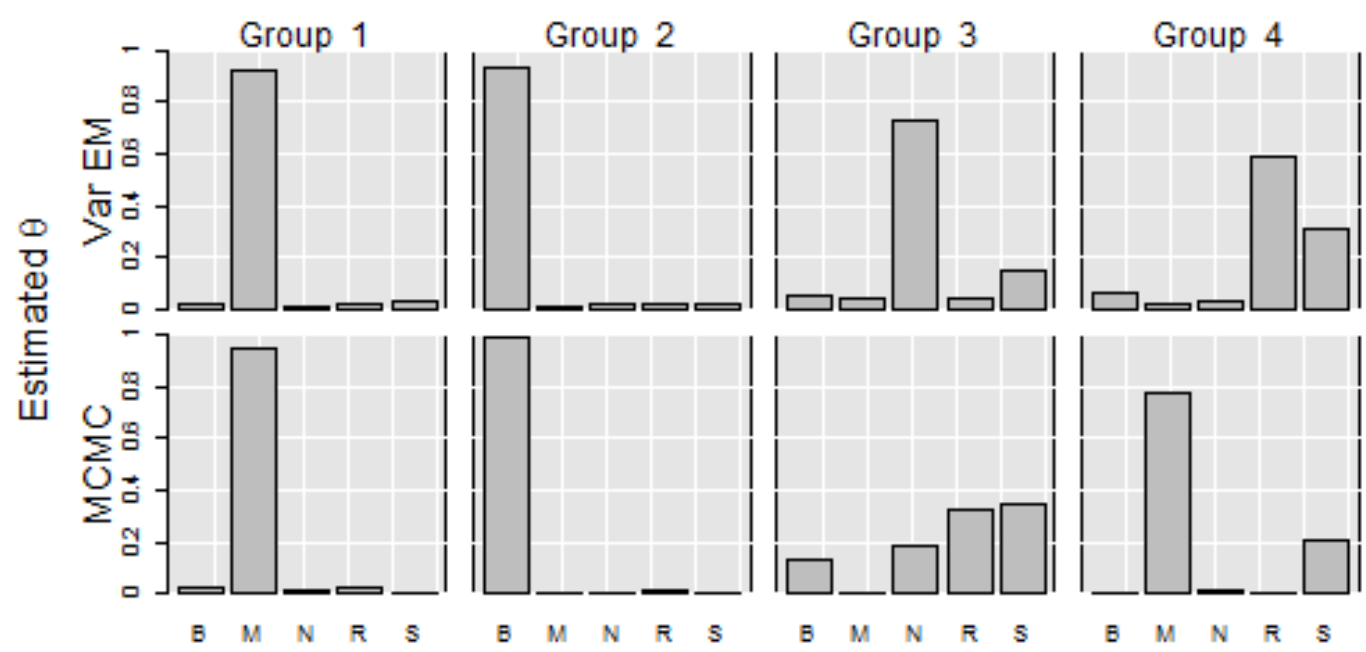

Figure 1: Estimated support parameters from the variational EM method and MCMC GM model

Table 3: Estimated $\alpha$ and relative frequency of each subgroup for random restart resulting in largest ELBO

\begin{tabular}{rrrrr}
\hline & Group 1 & Group 2 & Group 3 & Group 4 \\
\hline$\hat{\alpha}$ & 0.045 & 0.030 & 0.015 & 0.011 \\
Relative Freq & 0.45 & 0.30 & 0.15 & 0.11 \\
\hline
\end{tabular}

\section{Discussion}

When estimating the same parameters, a single variational EM procedure is up to 140 times faster than the MCMC procedure of Gormley and Murphy [2009]. Despite the multi-modality of the variational objective, we see that a single run is generally enough to find a reasonable point estimate. Furthermore, when we include estimation of the Dirichlet membership parameter $\alpha$, the goodness-of-fit is drastically improved and we see substantive changes in model interpretation. We conclude that the variational EM approach is an attractive alternative to an MCMC estimation procedure for fitting mixed membership models with ranked data.

\section{References}

Isobel Claire Gormley and Thomas Brendan Murphy. A grade of membership model for rank data. Bayesian Anal., 4(2):265-295, 2009. ISSN 1936-0975. doi: 10.1214/09-BA410. URL http://dx.doi.org/10.1214/09-BA410.

Michael Marsh and Paul Mitchell. How Ireland voted 199\%. Westview Press, 1999. 
Ayala Cohen and CL Mallows. Assessing goodness of fit of ranking models to data. The Statistician, pages 361-374, 1983.

Elena A. Erosheva, Stephen E. Fienberg, and Cyrille Joutard. Describing disability through individual-level mixture models for multivariate binary data. Ann. Appl. Stat., 1(2):502-537, 2007. ISSN 1932-6157. doi: 10.1214/07-AOAS126. URL http://dx.doi.org/10.1214/07-A0AS126. 